\title{
The Laplacian-Energy-Like Invariants of Three Types of Lattices
}

\author{
Zheng-Qing Chu, ${ }^{1}$ Jia-Bao Liu, ${ }^{1}$ and Xiao-Xin $\mathrm{Li}^{2}$ \\ ${ }^{1}$ Department of Public Courses, Anhui Xinhua University, Hefei, Anhui 230088, China \\ ${ }^{2}$ College of Mathematics and Computer, Chizhou University, Chizhou, Anhui 247000, China \\ Correspondence should be addressed to Jia-Bao Liu; liujiabaoad@163.com and Xiao-Xin Li; lxx@czu.edu.cn
}

Received 18 November 2015; Revised 4 March 2016; Accepted 9 March 2016

Academic Editor: Karoly Heberger

Copyright (C) 2016 Zheng-Qing Chu et al. This is an open access article distributed under the Creative Commons Attribution License, which permits unrestricted use, distribution, and reproduction in any medium, provided the original work is properly cited.

\begin{abstract}
This paper mainly studies the Laplacian-energy-like invariants of the modified hexagonal lattice, modified Union Jack lattice, and honeycomb lattice. By utilizing the tensor product of matrices and the diagonalization of block circulant matrices, we derive closedform formulas expressing the Laplacian-energy-like invariants of these lattices. In addition, we obtain explicit asymptotic values of these invariants with software-aided computations of some integrals.
\end{abstract}

\section{Introduction}

Molecular structure descriptors or topological indices are used for modelling information of molecules, including toxicologic, chemical, and other properties of chemical compounds in theoretical chemistry. Topological indices play a very important role in mathematical chemistry, especially in the quantitative structure-property relationship (QSPR) and quantitative structure activity relationship (QSAR). Many topological indices have been introduced and investigated by mathematicians, chemists, and biologists, which contain energy [1], the Laplacian-energy-like invariant [2-5], the Kirchhoff index [6-13], and so forth. The energy of the graph is an important invariant of the adjacency spectrum and is the sum of the absolute values of all the eigenvalues of a graph $G$, which is studied in chemistry and used to approximate the total electron energy of a molecule [1]. During researching the character of the conjugated carbon oxides, chemists found that the "general electric" $E_{\pi}$ is closely related to the energy releasing from the formation progress of the conjugated carbon oxides and could be approximately calculated by Hückel molecular orbital theory. And in the method of HMO, the calculation of $E_{\pi}$ can be attributed to the sum of the absolute values of all the eigenvalues of its molecular graph [14-20].

Compared with adjacency matrix, the definition of Laplacian matrix added to all vertices degrees. As Mohar said, the Laplacian eigenvalues can reflect more the combination properties of graphs. Cvetković and Simić [21-23] pointed out that, as molecular structure descriptors, the Laplacianenergy-like invariant not only well describes the properties of most of the descriptors which are indicated, such as entropy, molar volume, and molar refractivity, but also is able to describe some more difficult properties, such as boiling point and rub points. Due to the fact that Laplacian-energy-like invariant has a significant physical and chemical background $[24,25]$, it has received wide attention to research it from many mathematical and chemical workers.

All the graphs discussed in this paper are simple, finite, and undirected. For a graph $G$, the vertex set and edge set of $G$ will be denoted by $V(G)=\left\{v_{1}, v_{2}, \ldots, v_{n}\right\}$ and $E(G)=$ $\left\{e_{1}, e_{2}, \ldots, e_{m}\right\}$, respectively [26]. The adjacency matrix and the diagonal matrix of $G$ are, respectively, $A(G)$ and $D(G)$; then the matrix $L(G)=D(G)-A(G)$ is called the Laplacian matrix of the graph $G[27,28]$. The characteristic polynomials and Laplacian polynomials of the graph $G$ are $\chi_{G}(\lambda)=$ $\operatorname{det}(\lambda I-A(G))$ and $\mu_{G}(\lambda)=\operatorname{det}(\lambda I-L(G))$ [29]. Both $A(G)$ and $L(G)$ are symmetric matrices; their eigenvalues are real numbers $[30,31]$. Thus, we can order the eigenvalues of the graph $G$ as $\lambda_{1} \geq \lambda_{2} \geq \cdots \geq \lambda_{n}$, and the Laplacian eigenvalues are $\mu_{1} \geq \mu_{2} \geq \cdots \geq \mu_{n}[32,33]$. If $G$ is a connected graph, then $\mu_{i}>0, i=1,2, \ldots, n-1, \mu_{n}=0$ [34-36]. Next, we will recall some basic concepts. 
Definition 1 (see [1]). The energy of a graph $G$ is the sum of the absolute values of all the eigenvalues of $G$; that is,

$$
E(G)=\sum_{i=1}^{n}\left|\lambda_{i}\right|
$$

Definition 2 (see [2]). Let $G$ be a graph of order $n$. The Laplacian-energy-like invariant of $G$, denoted by $\operatorname{LEL}(G)$, is defined as

$$
\operatorname{LEL}(G)=\sum_{i=1}^{n} \sqrt{\mu_{i}} .
$$

Definition 3 (see [35]). For two matrices $A=\left(a_{i, j}\right)_{m \times n}, B=$ $\left(b_{i, j}\right)_{s \times t}$, the tensor product of $A$ and $B$, denoted by $A \otimes B$, is defined as

$$
\left(\begin{array}{cccc}
a_{11} B & a_{12} B & \cdots & a_{1 n} B \\
\cdots & \cdots & \cdots & \cdots \\
\cdots & \cdots & \cdots & \cdots \\
a_{m 1} B & a_{m 2} B & \cdots & a_{m n} B
\end{array}\right)
$$

Theorem 4 (see [35]). Let $\left\{G_{n}\right\}$ be a sequence of finite simple graphs with bounded average degree such that

$$
\begin{aligned}
& \lim _{n \rightarrow \infty}\left|V\left(G_{n}\right)\right|=\infty, \\
& \lim _{n \rightarrow \infty} \frac{L E L\left(G_{n}\right)}{\left|V\left(G_{n}\right)\right|}=h \neq 0 .
\end{aligned}
$$
that

Let $\left\{H_{n}\right\}$ be a sequence of spanning subgraphs of $\left\{G_{n}\right\}$ such

$$
\lim _{n \rightarrow \infty} \frac{\left|\left\{v \in V\left(H_{n}\right): d_{H_{n}(v)}=d_{G_{n}(v)}\right\}\right|}{\left|V\left(G_{n}\right)\right|}=1
$$

then

$$
\lim _{n \rightarrow \infty} \frac{\operatorname{LEL}\left(H_{n}\right)}{\left|V\left(G_{n}\right)\right|}=h
$$

That is, $G_{n}$ and $H_{n}$ have the same asymptotic Laplacianenergy-like invariant.

In what follows, we will explore the Laplacian-energylike invariants formulas of the modified hexagonal lattice, modified Union Jack lattice, and honeycomb lattice.

\section{Main Results}

2.1. The Laplacian-Energy-Like Invariant of the Modified Hexagonal Lattice. The modified hexagon lattice with toroidal boundary condition is denoted by $\operatorname{MH}^{t}\left(n_{1}, n_{2}\right)$.
Theorem 5. Let $\alpha_{i}=2 \pi i / n_{1}, \beta_{j}=2 \pi j / n_{2}$. Then

(1) $\operatorname{LEL}\left(M H^{t}\left(n_{1}, n_{2}\right)\right)$

$$
=\sum_{i=0}^{n_{1}-1} \sum_{j=0}^{n_{2}-1} \sqrt{6-2 \cos \alpha_{i}-2 \cos \beta_{j}-2 \cos \left(\alpha_{i}-\beta_{j}\right)},
$$

(2) $\lim _{n_{1} \rightarrow \infty} \lim _{n_{2} \rightarrow \infty} \frac{\operatorname{LEL}\left(M H^{t}\left(n_{1}, n_{2}\right)\right)}{n_{1} n_{2}}=\frac{1}{4 \pi^{2}}$

$\cdot \iint_{0}^{2 \pi} \sqrt{6-2 \cos x-2 \cos y-2 \cos (x-y)} d x d y$

$\approx 2.3705$.

Proof. With the proper labelling of the vertices of the modified hexagonal lattice, its Laplacian matrix is

$$
\begin{aligned}
L & \left(\mathrm{MH}^{t}\left(n_{1}, n_{2}\right)\right) \\
& =\left(\begin{array}{ccccccc}
C & -P & 0 & \cdots & 0 & 0 & -P^{T} \\
-P^{T} & C & -P & \cdots & 0 & 0 & 0 \\
0 & -P^{T} & C & \cdots & 0 & 0 & 0 \\
\cdots & \cdots & \cdots & \cdots & \cdots & \cdots & \cdots \\
0 & 0 & 0 & \cdots & C & -P & 0 \\
0 & 0 & 0 & \cdots & -P^{T} & C & -P \\
-P & 0 & 0 & \cdots & 0 & -P^{T} & C
\end{array}\right)_{n_{2} \times n_{2}}, \\
C & =\left(\begin{array}{ccccccc}
6 & -1 & 0 & \cdots & 0 & 0 & -1 \\
-1 & 6 & -1 & \cdots & 0 & 0 & 0 \\
0 & -1 & 6 & \cdots & 0 & 0 & 0 \\
\cdots & \cdots & \cdots & \cdots & \cdots & \cdots & \cdots \\
0 & 0 & 0 & \cdots & 6 & -1 & 0 \\
0 & 0 & 0 & \cdots & -1 & 6 & -1 \\
-1 & 0 & 0 & \cdots & 0 & -1 & 6
\end{array}\right)_{n_{1} \times n_{1}}, \\
P & =\left(\begin{array}{ccccccc}
1 & 0 & 0 & \cdots & 0 & 0 & 1 \\
1 & 1 & 0 & \cdots & 0 & 0 & 0 \\
0 & 1 & 1 & \cdots & 0 & 0 & 0 \\
\cdots & \cdots & \cdots & \cdots & \cdots & \cdots & \cdots \\
0 & 0 & 0 & \cdots & 1 & 0 & 0 \\
0 & 0 & 0 & \cdots & 1 & 1 & 0 \\
0 & 0 & 0 & \cdots & 0 & 1 & 1
\end{array}\right)_{n_{1} \times n_{1}},
\end{aligned}
$$

where $I_{n_{1}}, I_{n_{2}}$ are the unit matrices and $M \otimes N$ is tensor product of matrices $M$ and $N$. Consider

$$
B_{n_{2}}=\left(\begin{array}{cccccc}
0 & 1 & 0 & \cdots & 0 & 0 \\
0 & 0 & 1 & \cdots & 0 & 0 \\
\cdots & \cdots & \cdots & \cdots & \cdots & \cdots \\
0 & 0 & 0 & \cdots & 0 & 1 \\
1 & 0 & 0 & \cdots & 0 & 0
\end{array}\right)_{n_{2} \times n_{2}} .
$$


The matrix $L\left(\mathrm{MH}^{t}\left(n_{1}, n_{2}\right)\right)$ can be defined as follows:

$$
\begin{aligned}
L\left(\mathrm{MH}^{t}\left(n_{1}, n_{2}\right)\right)= & I_{n_{2}} \otimes C_{n_{1}}-B_{n_{2}} \otimes P_{n_{1}}-B_{n_{2}}^{T} \otimes P_{n_{1}}^{T} \\
= & I_{n_{2}} \otimes\left(6 I_{n_{1}}-B_{n_{1}}-B_{n_{1}}^{T}\right)-B_{n_{2}} \\
& \otimes\left(I_{n_{1}}+B_{n_{1}}^{T}\right)-B_{n_{2}}^{T} \\
& \otimes\left(I_{n_{1}}+B_{n_{1}}\right) .
\end{aligned}
$$

Let $\left\{1=g^{0}, g^{1}, \ldots, g^{n-1}\right\}$ be a cyclic group of order $n$. Obviously, $\rho: g^{i} \rightarrow B_{n}^{i}$ can express the group. The cyclic group of order $n$ has linear values of $n \chi_{i}(i=0,1, \ldots, n-$ $1), \chi_{i}(g)=\omega_{n}^{i}$, where $\omega_{n}$ are said $n$-times unit roots.

Therefore, there is a reversible matrix

$$
Q_{n}=\left(\frac{\omega_{n}^{i j}}{\sqrt{n}}\right)_{0 \leq i, j \leq n-1},
$$

such that

$$
Q_{n}^{-1} B_{n} Q_{n}=\operatorname{diag}\left(1, \omega_{n}, \ldots, \omega_{n}^{n-1}\right)=: D_{n} .
$$

In fact,

$$
\begin{aligned}
B_{n}^{T} & =B_{n}^{-1}, \\
Q_{n}^{T} & =Q_{n}^{-1} ;
\end{aligned}
$$

hence

$$
Q_{n}^{-1} B_{n}^{T} Q_{n}=\operatorname{diag}\left(1, \omega^{-1}, \ldots, \omega_{n}^{-(n-1)}\right)=: D_{n}^{-1} .
$$

So

$$
\begin{aligned}
& \left(Q_{n_{2}}^{-1} \otimes Q_{n_{1}}^{-1}\right) L\left(\mathrm{MH}^{t}\left(n_{1}, n_{2}\right)\right)\left(Q_{n_{2}} \otimes Q_{n_{1}}\right)=\left(Q_{n_{2}}^{-1}\right. \\
& \left.\quad \otimes Q_{n_{1}}^{-1}\right)\left[I_{n_{2}} \otimes\left(6 I_{n_{1}}-B_{n_{1}}-B_{n_{1}}^{T}\right)-B_{n_{2}}\right. \\
& \left.\quad \otimes\left(I_{n_{1}}+B_{n_{1}}^{T}\right)-B_{n_{2}}^{T} \otimes\left(I_{n_{1}}+B_{n_{1}}\right)\right]\left(Q_{n_{2}} \otimes Q_{n_{1}}\right) \\
& \quad=I_{n_{2}} \otimes\left(6 I_{n_{1}}-D_{n_{1}}-D_{n_{1}}^{-1}\right)-D_{n_{2}} \otimes\left(I_{n_{1}}+D_{n_{1}}^{-1}\right) \\
& \quad-D_{n_{2}}^{-1} \otimes\left(I_{n_{1}}+D_{n_{1}}\right) .
\end{aligned}
$$

It is not difficult to find that $I_{n_{2}} \otimes\left(6 I_{n_{1}}-D_{n_{1}}-D_{n_{1}}^{-1}\right)-$ $D_{n_{2}} \otimes\left(I_{n_{1}}+D_{n_{1}}^{-1}\right)-D_{n_{2}}^{-1} \otimes\left(I_{n_{1}}+D_{n_{1}}\right)$ is a diagonal matrix whose diagonal elements are

$$
\begin{aligned}
6- & \omega_{n_{1}}^{i}-\omega_{n_{1}}^{-i}-\omega_{n_{2}}^{j}-\omega_{n_{2}}^{-j}-\left(\omega_{n_{1}}^{i} \omega_{n_{2}}^{-j}+\omega_{n_{1}}^{-i} \omega_{n_{2}}^{j}\right) \\
= & 6-2 \cos \frac{2 i \pi}{n_{1}}-2 \cos \frac{2 j \pi}{n_{2}} \\
& -2\left(\cos \frac{2 i \pi}{n_{1}}-\cos \frac{2 j \pi}{n_{2}}\right),
\end{aligned}
$$

where $0 \leq i \leq n_{1}-1$ and $0 \leq j \leq n_{2}-1$.

This means that the eigenvalues of the matrix $L$ are $\mu=$ $6-2 \cos \alpha_{i}-2 \cos \beta_{j}-2 \cos \left(\alpha_{i}-\beta_{j}\right), 0 \leq i \leq n_{1}-1$, and $0 \leq$ $j \leq n_{2}-1$, where $\alpha_{i}=2 \pi i / n_{1}$ and $\beta_{j}=2 \pi j / n_{2}$.
By formula (2), the Laplacian-energy-like invariant is

$$
\begin{aligned}
& \operatorname{LEL}\left(\operatorname{MH}^{t}\left(n_{1}, n_{2}\right)\right) \\
& \quad=\sum_{i=0}^{n_{1}-1} \sum_{j=0}^{n_{2}-1} \sqrt{6-2 \cos \alpha_{i}-2 \cos \beta_{j}-2 \cos \left(\alpha_{i}-\beta_{j}\right)} . \\
& \text { So } \\
& \lim _{n_{1} \rightarrow \infty} \lim _{n_{2} \rightarrow \infty} \frac{\operatorname{LEL}\left(\mathrm{MH}^{t}\left(n_{1}, n_{2}\right)\right)}{n_{1} n_{2}}=\lim _{n_{1} \rightarrow \infty} \lim _{n_{2} \rightarrow \infty} \frac{1}{n_{1} n_{2}} \\
& \quad \cdot \sum_{i=0}^{n_{1}-1} \sum_{j=0}^{n_{2}-1} \sqrt{6-2 \cos \alpha_{i}-2 \cos \beta_{j}-2 \cos \left(\alpha_{i}-\beta_{j}\right)} \\
& \quad=\iint_{0}^{1} \sqrt{6-2 \cos 2 \pi x-2 \cos 2 \pi y-2 \cos 2 \pi(x-y)} d x d y \\
& =\frac{1}{4 \pi^{2}} \iint_{0}^{2 \pi} \sqrt{6-2 \cos x-2 \cos y-2 \cos (x-y)} d x d y \\
& \approx 2.3705 .
\end{aligned}
$$

Remark 6. The numerical integration value in last line is calculated with the software MATLAB [37]. As such computations would be possible on a computer with high memory and processing speed, we used Mac Pro with processor $2 \times$ $2.93 \mathrm{GHz} 6$-core Intel Xeon (24 hyperthreads in total) and memory $24 \mathrm{~GB} 1333 \mathrm{MHz}$ DDR3 to obtain the results.

By Theorems 4 and 5, we can immediately arrive at the following theorem.

Theorem 7. For the modified hexagonal lattices $M H^{t}\left(n_{1}, n_{2}\right)$, $M H^{c}\left(n_{1}, n_{2}\right)$, and $M H^{f}\left(n_{1}, n_{2}\right)$ with toroidal, cylindrical, and free boundary conditions, then,

$$
\begin{aligned}
\text { (1) } & \lim _{n_{1} \rightarrow \infty} \lim _{n_{2} \rightarrow \infty} \frac{\operatorname{LEL}\left(M H^{t}\left(n_{1}, n_{2}\right)\right)}{n_{1} n_{2}} \\
= & \lim _{n_{1} \rightarrow \infty} \lim _{n_{2} \rightarrow \infty} \frac{\operatorname{LEL}\left(M H^{c}\left(n_{1}, n_{2}\right)\right)}{n_{1} n_{2}} \\
= & \lim _{n_{1} \rightarrow \infty} \lim _{n_{2} \rightarrow \infty} \frac{\operatorname{LEL}\left(M H^{f}\left(n_{1}, n_{2}\right)\right)}{n_{1} n_{2}} \approx 2.3705, \\
\text { (2) } & \operatorname{LEL}\left(M H^{t}\left(n_{1}, n_{2}\right)\right)=\operatorname{LEL}\left(M H^{c}\left(n_{1}, n_{2}\right)\right) \\
= & \operatorname{LEL}\left(M H^{f}\left(n_{1}, n_{2}\right)\right) \approx 2.3705 n_{1} n_{2} .
\end{aligned}
$$

2.2. The Laplacian-Energy-Like Invariant of the Modified Union Jack Lattice. The modified Union Jack lattice with toroidal boundary condition is denoted by $S^{t}\left(n_{1}, n_{2}\right)$.

Theorem 8. Let $\alpha_{i}=2 \pi i / n_{1} ; \beta_{j}=2 \pi j / n_{2}$. Then

(1) $\operatorname{LEL}\left(S^{t}\left(n_{1}, n_{2}\right)\right)$

$$
=\sum_{i=0}^{n_{1}-1} \sum_{j=0}^{n_{2}-1} \sqrt{8-2 \cos \alpha_{i}-2 \cos \beta_{j}-4 \cos \alpha_{i} \cos \beta_{j}},
$$


(2) $\lim _{n_{1} \rightarrow \infty} \lim _{n_{2} \rightarrow \infty} \frac{\operatorname{LEL}\left(S^{t}\left(n_{1}, n_{2}\right)\right)}{n_{1} n_{2}}=\frac{1}{4 \pi^{2}}$

$\iint_{0}^{2 \pi} \sqrt{8-2 \cos x-2 \cos y-4 \cos x \cos y} d x d y$

$\approx 2.7586$.

Proof. With a proper labelling of the vertices of the modified Union Jack lattice, its Laplacian matrix can be represented as

$$
L\left(S^{t}\left(n_{1}, n_{2}\right)\right)
$$$$
=\left(\begin{array}{ccccccc}
G & -U & 0 & \cdots & 0 & 0 & -U^{T} \\
-U^{T} & G & -U & \cdots & 0 & 0 & 0 \\
0 & -U^{T} & G & \cdots & 0 & 0 & 0 \\
\cdots & \cdots & \cdots & \cdots & \cdots & \cdots & \cdots \\
0 & 0 & 0 & \cdots & G & -U & 0 \\
0 & 0 & 0 & \cdots & -U^{T} & G & -U \\
-U & 0 & 0 & \cdots & 0 & -U^{T} & G
\end{array}\right)_{n_{2} \times n_{2}}
$$$$
G=\left(\begin{array}{ccccccc}
8 & -1 & 0 & \cdots & 0 & 0 & -1 \\
-1 & 8 & -1 & \cdots & 0 & 0 & 0 \\
0 & -1 & 8 & \cdots & 0 & 0 & 0 \\
\cdots & \cdots & \cdots & \cdots & \cdots & \cdots & \cdots \\
0 & 0 & 0 & \cdots & 8 & -1 & 0 \\
0 & 0 & 0 & \cdots & -1 & 8 & -1 \\
-1 & 0 & 0 & \cdots & 0 & -1 & 8
\end{array}\right)
$$$$
U=\left(\begin{array}{ccccccc}
1 & 1 & 0 & \cdots & 0 & 0 & 1 \\
1 & 1 & 1 & \cdots & 0 & 0 & 0 \\
0 & 1 & 1 & \cdots & 0 & 0 & 0 \\
\cdots & \cdots & \cdots & \cdots & \cdots & \cdots & \cdots \\
0 & 0 & 0 & \cdots & 1 & 1 & 0 \\
0 & 0 & 0 & \cdots & 1 & 1 & 1 \\
1 & 0 & 0 & \cdots & 0 & 1 & 1
\end{array}\right)
$$

Based on Theorem 5, we get

$$
\begin{aligned}
L\left(S^{t}\left(n_{1}, n_{2}\right)\right)= & I_{n_{2}} \otimes G_{n_{1}}-B_{n_{2}} \otimes U_{n_{1}}-B_{n_{2}}^{T} \otimes U_{n_{1}}^{T} \\
= & I_{n_{2}} \otimes\left(8 I_{n_{1}}-B_{n_{1}}-B_{n_{1}}^{T}\right)-B_{n_{2}} \\
& \otimes\left(I_{n_{1}}+B_{n_{1}}+B_{n_{1}}^{T}\right)-B_{n_{2}}^{T} \\
& \otimes\left(I_{n_{1}}+B_{n_{1}}^{T}+B_{n_{1}}\right) .
\end{aligned}
$$

Let

$$
Q_{n}=\left(\frac{\omega_{n}^{i j}}{\sqrt{n}}\right)_{0 \leq i, j \leq n-1},
$$

such that

$$
Q_{n}^{-1} B_{n} Q_{n}=\operatorname{diag}\left(1, \omega_{n}, \ldots, \omega_{n}^{n-1}\right)=: D_{n} .
$$

Actually,

$$
\begin{aligned}
B_{n}^{T} & =B_{n}^{-1}, \\
Q_{n}^{T} & =Q_{n}^{-1} ;
\end{aligned}
$$

consequently,

$$
Q_{n}^{-1} B_{n}^{T} Q_{n}=\operatorname{diag}\left(1, \omega^{-1}, \ldots, \omega_{n}^{-(n-1)}\right)=: D_{n}^{-1} .
$$

So

$$
\begin{aligned}
& \left(Q_{n_{2}}^{-1} \otimes Q_{n_{1}}^{-1}\right) L\left(S^{t}\left(n_{1}, n_{2}\right)\right)\left(Q_{n_{2}} \otimes Q_{n_{1}}\right)=\left(Q_{n_{2}}^{-1}\right. \\
& \left.\otimes Q_{n_{1}}^{-1}\right)\left[I_{n_{2}} \otimes\left(8 I_{n_{1}}-B_{n_{1}}-B_{n_{1}}^{T}\right)-B_{n_{2}}\right. \\
& \left.\otimes\left(I_{n_{1}}+B_{n_{1}}+B_{n_{1}}^{T}\right)-B_{n_{2}}^{T} \otimes\left(I_{n_{1}}+B_{n_{1}}^{T}+B_{n_{1}}\right)\right]\left(Q_{n_{2}}\right. \\
& \left.\otimes Q_{n_{1}}\right)=I_{n_{2}} \otimes\left(8 I_{n_{1}}-D_{n_{1}}-D_{n_{1}}^{-1}\right)-D_{n_{2}} \otimes\left(I_{n_{1}}\right. \\
& \left.+D_{n_{1}}+D_{n_{1}}^{-1}\right)-D_{n_{2}}^{-1} \otimes\left(I_{n_{1}}+D_{n_{1}}^{-1}+D_{n_{1}}\right) .
\end{aligned}
$$

It is not difficult to find that

$$
\begin{aligned}
I_{n_{2}} & \otimes\left(8 I_{n_{1}}-D_{n_{1}}-D_{n_{1}}^{-1}\right)-D_{n_{2}} \otimes\left(I_{n_{1}}+D_{n_{1}}+D_{n_{1}}^{-1}\right) \\
& -D_{n_{2}}^{-1} \otimes\left(I_{n_{1}}+D_{n_{1}}^{-1}+D_{n_{1}}\right)
\end{aligned}
$$

is a diagonal matrix whose diagonal elements are

$$
\begin{aligned}
8- & \omega_{n_{1}}^{i}-\omega_{n_{1}}^{-i}-\omega_{n_{2}}^{j}-\omega_{n_{2}}^{-j} \\
& -\left(\omega_{n_{1}}^{i} \omega_{n_{2}}^{j}+\omega_{n_{1}}^{i} \omega_{n_{2}}^{-j}+\omega_{n_{1}}^{-i} \omega_{n_{2}}^{-j}+\omega_{n_{1}}^{-i} \omega_{n_{2}}^{j}\right) \\
= & 8-2 \cos \frac{2 i \pi}{n_{1}}-2 \cos \frac{2 j \pi}{n_{2}}-4 \cos \frac{2 i \pi}{n_{1}} \cos \frac{2 j \pi}{n_{2}},
\end{aligned}
$$

where $0 \leq i \leq n_{1}-1$ and $0 \leq j \leq n_{2}-1$.

This means that the eigenvalues of the matrix $L$ are $\mu=$ $8-2 \cos \alpha_{i}-2 \cos \beta_{j}-4 \cos \alpha_{i} \beta_{j}, 0 \leq i \leq n_{1}-1$, and $0 \leq j \leq$ $n_{2}-1$, where $\alpha_{i}=2 \pi i / n_{1}$ and $\beta_{j}=2 \pi j / n_{2}$.

By formula (2), the Laplacian-energy-like invariant is

$$
\begin{aligned}
\operatorname{LEL} & \left(S^{t}\left(n_{1}, n_{2}\right)\right) \\
= & \sum_{i=0}^{n_{1}-1} \sum_{j=0}^{n_{2}-1} \sqrt{8-2 \cos \alpha_{i}-2 \cos \beta_{j}-4 \cos \alpha_{i} \cos \beta_{j}} .
\end{aligned}
$$

So

$\lim _{n_{1} \rightarrow \infty} \lim _{n_{2} \rightarrow \infty} \frac{\operatorname{LEL}\left(S^{t}\left(n_{1}, n_{2}\right)\right)}{n_{1} n_{2}}=\lim _{n_{1} \rightarrow \infty} \lim _{n_{2} \rightarrow \infty} \frac{1}{n_{1} n_{2}}$

$$
\begin{aligned}
& \cdot \sum_{i=0}^{n_{1}-1} \sum_{j=0}^{n_{2}-1} \sqrt{8-2 \cos \alpha_{i}-2 \cos \beta_{j}-4 \cos \alpha_{i} \cos \beta_{j}} \\
& =\iint_{0}^{1} \sqrt{8-2 \cos 2 \pi x-2 \cos 2 \pi y-4 \cos 2 \pi x \cos 2 \pi y} d x d y \\
& =\frac{1}{4 \pi^{2}} \iint_{0}^{2 \pi} \sqrt{8-2 \cos x-2 \cos y-4 \cos x \cos y} d x d y \\
& \approx 2.7586 .
\end{aligned}
$$


By Theorems 4 and 8 , it is not difficult to arrive at the following theorem.

Theorem 9. For the modified Union Jack lattices $S^{t}\left(n_{1}, n_{2}\right)$, $S^{c}\left(n_{1}, n_{2}\right)$, and $S^{f}\left(n_{1}, n_{2}\right)$ with toroidal, cylindrical, and free boundary conditions, then,

$$
\begin{aligned}
\text { (1) } & \lim _{n_{1} \rightarrow \infty} \lim _{n_{2} \rightarrow \infty} \frac{\operatorname{LEL}\left(S^{t}\left(n_{1}, n_{2}\right)\right)}{n_{1} n_{2}} \\
= & \lim _{n_{1} \rightarrow \infty} \lim _{n_{2} \rightarrow \infty} \frac{\operatorname{LEL}\left(S^{c}\left(n_{1}, n_{2}\right)\right)}{n_{1} n_{2}} \\
= & \lim _{n_{1} \rightarrow \infty} \lim _{n_{2} \rightarrow \infty} \frac{\operatorname{LEL}\left(S^{f}\left(n_{1}, n_{2}\right)\right)}{n_{1} n_{2}} \approx 2.7586, \\
\text { (2) } & \operatorname{LEL}\left(S^{t}\left(n_{1}, n_{2}\right)\right)=\operatorname{LEL}\left(S^{c}\left(n_{1}, n_{2}\right)\right) \\
= & \operatorname{LEL}\left(S^{f}\left(n_{1}, n_{2}\right)\right) \approx 2.7586 n_{1} n_{2} .
\end{aligned}
$$

2.3. The Laplacian-Energy-Like Invariant of the Honeycomb Lattice. The honeycomb lattice with toroidal boundary condition, denoted by $\operatorname{HC}^{t}\left(n_{1}, n_{2}\right)$, can be constructed by starting with an $m \times n$ square lattice and adding two diagonal edges to each square.

Theorem 10. Let $\alpha_{i}=2 \pi i / n_{1}$ and $\beta_{j}=2 \pi j / n_{2}$. Then

(1) $\operatorname{LEL}\left(H C^{t}\left(n_{1}, n_{2}\right)\right)$

$$
\begin{aligned}
& =\sum_{i=0}^{n_{1}-1} \sum_{j=0}^{n_{2}-1} \sqrt{3+\sqrt{3+2 \cos \alpha_{i}+2 \cos \beta_{j}+2 \cos \left(\alpha_{i}-\beta_{j}\right)}} \\
& +\sum_{i=0}^{n_{1}-1} \sum_{j=0}^{n_{2}-1} \sqrt{3-\sqrt{3+2 \cos \alpha_{i}+2 \cos \beta_{j}+2 \cos \left(\alpha_{i}-\beta_{j}\right)}}, \\
& \text { (2) } \lim _{n_{1} \rightarrow \infty} \lim _{n_{2} \rightarrow \infty} \frac{L E L\left(H C^{t}\left(n_{1}, n_{2}\right)\right)}{2 n_{1} n_{2}}=\frac{1}{8 \pi^{2}} \\
& \cdot \iint_{0}^{2 \pi} \sqrt{3+\sqrt{3+2 \cos x+2 \cos y+2 \cos (x-y)}} d x d y \\
& +\frac{1}{8 \pi^{2}} \\
& \cdot \iint_{0}^{2 \pi} \sqrt{3-\sqrt{3+2 \cos x+2 \cos y+2 \cos (x-y)}} d x d y \\
& \approx 1.6357 .
\end{aligned}
$$

Proof. Similarly, the Laplacian matrix of the honeycomb lattice is $L\left(\operatorname{HC}^{t}\left(n_{1}, n_{2}\right)\right)=\left(\begin{array}{cc}3 I_{M} & -F \\ -F^{T} & 3 I_{M}\end{array}\right)$, where $M=n_{1} n_{2}$ and $F$ is an $M \times M$ matrix. The matrix $F$ can be written in the following form:

$$
F=\left(\begin{array}{ccccccc}
W & 0 & 0 & \cdots & 0 & I & I \\
I & W & 0 & \cdots & 0 & 0 & 0 \\
0 & I & W & \cdots & 0 & 0 & 0 \\
\cdots & \cdots & \cdots & \cdots & \cdots & \cdots & \cdots \\
0 & 0 & 0 & \cdots & W & 0 & 0 \\
0 & 0 & 0 & \cdots & I & W & 0 \\
0 & 0 & 0 & \cdots & 0 & I & W
\end{array}\right)_{n_{2} \times n_{2}},
$$

$$
W=\left(\begin{array}{ccccccc}
1 & 0 & 0 & \cdots & 0 & 0 & 1 \\
1 & 1 & 0 & \cdots & 0 & 0 & 0 \\
0 & 1 & 1 & \cdots & 0 & 0 & 0 \\
\cdots & \cdots & \cdots & \cdots & \cdots & \cdots & \cdots \\
0 & 0 & 0 & \cdots & 1 & 0 & 0 \\
0 & 0 & 0 & \cdots & 1 & 1 & 0 \\
1 & 0 & 0 & \cdots & 0 & 1 & 1
\end{array}\right)_{n_{1} \times n_{1}}
$$

where $I$ represents the unit matrix of $n_{1} \times n_{1}$ and $I_{M}$ represents the unit matrix of $M \times M$, respectively.

Based on Theorem 5, the matrix $F$ can be written as

$$
\begin{aligned}
F & =I_{n_{2}} \otimes W_{n_{1}}+B_{n_{2}}^{T} \otimes I_{n_{1}} \\
& =I_{n_{2}} \otimes\left(I_{n_{1}}+B_{n_{1}}^{T}\right)+B_{n_{2}}^{T} \otimes I_{n_{1}} .
\end{aligned}
$$

Let

$$
Q_{n}=\left(\frac{\omega_{n}^{i j}}{\sqrt{n}}\right)_{0 \leq i, j \leq n-1},
$$

such that

$$
Q_{n}^{-1} B_{n} Q_{n}=\operatorname{diag}\left(1, \omega_{n}, \ldots, \omega_{n}^{n-1}\right)=: D_{n} .
$$

Similarly,

$$
\begin{aligned}
B_{n}^{T} & =B_{n}^{-1}, \\
Q_{n}^{T} & =Q_{n}^{-1} ;
\end{aligned}
$$

hence,

$$
Q_{n}^{-1} B_{n}^{T} Q_{n}=\operatorname{diag}\left(1, \omega^{-1}, \ldots, \omega_{n}^{-(n-1)}\right)=: D_{n}^{-1} .
$$

So

$$
\begin{aligned}
& \left(Q_{n_{2}}^{-1} \otimes Q_{n_{1}}^{-1}\right) F\left(Q_{n_{2}} \otimes Q_{n_{1}}\right)=\left(Q_{n_{2}}^{-1} \otimes Q_{n_{1}}^{-1}\right) \\
& \quad \cdot\left[I_{n_{2}} \otimes\left(I_{n_{1}}+B_{n_{1}}^{T}\right)+B_{n_{2}}^{T} \otimes I_{n_{1}}\right]\left(Q_{n_{2}} \otimes Q_{n_{1}}\right)=I_{n_{2}} \\
& \otimes\left(I_{n_{1}}+D_{n_{1}}^{-1}\right)+D_{n_{2}}^{-1} \otimes I_{n_{1}} .
\end{aligned}
$$

It is not difficult to find that $I_{n_{2}} \otimes\left(I_{n_{1}}+D_{n_{1}}^{-1}\right)+D_{n_{2}}^{-1} \otimes I_{n_{1}}$ is a diagonal matrix whose diagonal elements are $1+\omega_{n_{1}}^{-i}+\omega_{n_{2}}^{-j}$, so matrix $L\left(\operatorname{HC}^{t}\left(n_{1}, n_{2}\right)\right)$ can be reduced to the following form:

$$
\begin{aligned}
L & \left(\operatorname{HC}^{t}\left(n_{1}, n_{2}\right)\right) \\
& =\left(\begin{array}{cc}
3 & -1-\omega_{n_{1}}^{-i}-\omega_{n_{2}}^{-j} \\
-1-\omega_{n_{1}}^{-i}-\omega_{n_{2}}^{-j} & 3
\end{array}\right) .
\end{aligned}
$$


$\operatorname{By} \operatorname{det}\left(\mu I-L\left(\operatorname{HC}^{t}\left(n_{1}, n_{2}\right)\right)\right)=0$, we can get

$$
\begin{aligned}
(\mu-3)^{2}= & \left(-1-\omega_{n_{1}}^{-i}-\omega_{n_{2}}^{-j}\right)\left(-1-\omega_{n_{1}}^{-i}-\omega_{n_{2}}^{-j}\right) \\
= & 3+\omega_{n_{1}}^{i}+\omega_{n_{1}}^{-i}+\omega_{n_{2}}^{j}+\omega_{n_{2}}^{-j}+\omega_{n_{1}}^{-i} \omega_{n_{2}}^{j} \\
& +\omega_{n_{1}}^{i} \omega_{n_{2}}^{-j} \\
= & 3+2 \cos \frac{2 \pi i}{n_{1}}+2 \cos \frac{2 \pi j}{n_{2}} \\
& +2 \cos \left(\cos \frac{2 \pi i}{n_{1}}-\cos \frac{2 \pi j}{n_{2}}\right) .
\end{aligned}
$$

Therefore, the $L\left(\operatorname{HC}^{t}\left(n_{1}, n_{2}\right)\right)$ characteristic eigenvalues are $\mu=3$

$$
\pm \sqrt{3+2 \cos \frac{2 \pi i}{n_{1}}+2 \cos \frac{2 \pi j}{n_{2}}+2 \cos \left(\frac{2 \pi i}{n_{1}}-\frac{2 \pi j}{n_{2}}\right)}
$$

where $0 \leq i \leq n_{1}-1$ and $0 \leq j \leq n_{2}-1$.

Let $\alpha_{i}=2 \pi i / n_{1}$ and $\beta_{j}=2 \pi j / n_{2}$. By formula (2), we may obtain the Laplacian-energy-like invariant:

$$
\begin{aligned}
\operatorname{LEL} & \left(\operatorname{HC}^{t}\left(n_{1}, n_{2}\right)\right) \\
= & \sum_{i=0}^{n_{1}-1} \sum_{j=0}^{n_{2}-1} \sqrt{3+\sqrt{3+2 \cos \alpha_{i}+2 \cos \beta_{j}+2 \cos \left(\alpha_{i}-\beta_{j}\right)}} \\
& +\sum_{i=0}^{n_{1}-1} \sum_{j=0}^{n_{2}-1} \sqrt{3-\sqrt{3+2 \cos \alpha_{i}+2 \cos \beta_{j}+2 \cos \left(\alpha_{i}-\beta_{j}\right)}} .
\end{aligned}
$$

By the definition of double integration, we arrive at

$$
\begin{aligned}
\lim _{n_{1} \rightarrow \infty} \lim _{n_{2} \rightarrow \infty} \frac{\operatorname{LEL}\left(\mathrm{HC}^{t}\left(n_{1}, n_{2}\right)\right)}{2 n_{1} n_{2}=} & \lim _{n_{1} \rightarrow \infty} \lim _{n_{2} \rightarrow \infty} \frac{1}{2 n_{1} n_{2}} \sum_{i=0}^{n_{1}-1} \sum_{j=0}^{n_{2}-1} \sqrt{3+\sqrt{3+2 \cos \alpha_{i}+2 \cos \beta_{j}+2 \cos \left(\alpha_{i}-\beta_{j}\right)}} \\
& +\lim _{n_{1} \rightarrow \infty} \lim _{n_{2} \rightarrow \infty} \frac{1}{2 n_{1} n_{2}} \sum_{i=0}^{n_{1}-1} \sum_{j=0}^{n_{2}-1} \sqrt{3-\sqrt{3+2 \cos \alpha_{i}+2 \cos \beta_{j}+2 \cos \left(\alpha_{i}-\beta_{j}\right)}} \\
= & \frac{1}{2} \iint_{0}^{1} \sqrt{3+\sqrt{3+2 \cos 2 \pi x+2 \cos 2 \pi y+2 \cos 2 \pi(x-y)}} d x d y \\
& +\frac{1}{2} \iint_{0}^{1} \sqrt{3-\sqrt{3+2 \cos 2 \pi x+2 \cos 2 \pi y+2 \cos 2 \pi(x-y)}} d x d y \\
= & \frac{1}{8 \pi^{2}} \iint_{0}^{2 \pi} \sqrt{3+\sqrt{3+2 \cos x+2 \cos y+2 \cos (x-y)}} d x d y \\
& +\frac{1}{8 \pi^{2}} \iint_{0}^{2 \pi} \sqrt{3-\sqrt{3+2 \cos x+2 \cos y+2 \cos (x-y)}} d x d y \approx 1.6357 .
\end{aligned}
$$

By Theorems 4 and 10, we can easily obtain the following theorem.

Theorem 11. For the honeycomb lattices $\operatorname{HC}^{t}\left(n_{1}, n_{2}\right)$, $H C^{c}\left(n_{1}, n_{2}\right)$, and $H C^{f}\left(n_{1}, n_{2}\right)$ with toroidal, cylindrical, and free boundary conditions, then,

$$
\text { (1) } \begin{aligned}
& \lim _{n_{1} \rightarrow \infty} \lim _{n_{2} \rightarrow \infty} \frac{\operatorname{LEL}\left(H C^{t}\left(n_{1}, n_{2}\right)\right)}{n_{1} n_{2}} \\
= & \lim _{n_{1} \rightarrow \infty} \lim _{n_{2} \rightarrow \infty} \frac{\operatorname{LEL}\left(H C^{c}\left(n_{1}, n_{2}\right)\right)}{n_{1} n_{2}} \\
= & \lim _{n_{1} \rightarrow \infty} \lim _{n_{2} \rightarrow \infty} \frac{\operatorname{LEL}\left(H C^{f}\left(n_{1}, n_{2}\right)\right)}{n_{1} n_{2}} \approx 1.6357 ;
\end{aligned}
$$

$$
\text { (2) } \begin{aligned}
\operatorname{LEL}\left(H C^{t}\left(n_{1}, n_{2}\right)\right)=\operatorname{LEL}\left(H C^{c}\left(n_{1}, n_{2}\right)\right) \\
=\operatorname{LEL}\left(H C^{f}\left(n_{1}, n_{2}\right)\right) \approx 1.6357 n_{1} n_{2} .
\end{aligned}
$$

\section{Conclusions}

In this paper, we mainly studied the Laplacian-energylike invariants of the modified hexagonal lattice, modified Jack lattice, and honeycomb lattice. The Laplacian-energylike invariants formulas of these lattices are obtained. The proposed results imply that the asymptotic Laplacian-energylike invariants of those lattices are independent of the three boundary conditions.

The problems on the various topological indices of lattices have much important significance in the mathematical 
theory, chemical energy, statistical physics, and networks science. This paper investigated the Laplacian-energy-like invariants of some lattices. However, the other topological indices of the general lattices remain to be studied.

\section{Competing Interests}

The authors declare that there is no conflict of interests regarding the publication of this paper.

\section{Acknowledgments}

The authors would like to express their sincere gratitude to the Natural Science Foundation for the Higher Education Institutions of Anhui Province of China (nos. KJ2013A196, KJ2013B105, and KJ2015A331), the key project of the Outstanding Young Talent Support Program of the University of Anhui Province (gxyqZD2016367), Anhui Provincial Natural Science Foundation (no. 1408085QA03), NSF of Department of Education of Anhui Province (KJ2015ZD27), and Quality Engineering Projects of Anhui Province of China under Grant no. 2014msgzs168.

\section{References}

[1] I. Gutman, “The energy of agraph," Ber. Math. Stat. Sekt. Forschungsz. Graz, vol. 103, pp. 1-22, 1978.

[2] J. Liu and B. Liu, "A Laplacian-energy-like invariant of a graph," MATCH Communications in Mathematical and in Computer Chemistry, vol. 59, pp. 355-372, 2008.

[3] B. Liu, Y. Huang, and Z. You, "A survey on the Laplacian-energylike invariant," MATCH Communications in Mathematical and in Computer Chemistry, vol. 66, no. 3, pp. 713-730, 2011.

[4] W. Wang, "Asymptotic behavior of Laplacian-energy-like invariant of some graphs," Ars Combinatoria, vol. 115, pp. 273282, 2014.

[5] W. Wang and Y. Luo, "On Laplacian-energy-like invariant of a graph," Linear Algebra and Its Applications, vol. 437, no. 2, pp. 713-721, 2012.

[6] L. H. Feng, G. Yu, K. Xu, and Z. Jiang, "A note on the Kirchhoff index of bicyclic graphs," Ars Combinatoria, vol. 114, pp. 33-40, 2014.

[7] J.-B. Liu and J. Cao, "The resistance distances of electrical networks based on Laplacian generalized inverse," Neurocomputing, vol. 167, pp. 306-313, 2015.

[8] J.-B. Liu, X.-F. Pan, L. Yu, and D. Li, "Complete characterization of bicyclic graphs with minimal Kirchhoff index," Discrete Applied Mathematics, vol. 200, pp. 95-107, 2016.

[9] F. Zhang, Y.-C. Chen, and Z. Chen, "Clique-inserted-graphs and spectral dynamics of clique-inserting," Journal of Mathematical Analysis and Applications, vol. 349, no. 1, pp. 211-225, 2009.

[10] J. Liu, J. Cao, X.-F. Pan, and A. Elaiw, "The Kirchhoff index of hypercubes and related complex networks," Discrete Dynamics in Nature and Society, vol. 2013, Article ID 543189, 7 pages, 2013.

[11] J.-B. Liu, X.-F. Pan, J. Cao, and F.-T. Hu, “The Kirchhoff index of some combinatorial networks," Discrete Dynamics in Nature and Society, vol. 2015, Article ID 340793, 6 pages, 2015.

[12] J. Liu, X.-F. Pan, Y. Wang, and J. Cao, "The Kirchhoff index of folded hypercubes and some variant networks," Mathematical
Problems in Engineering, vol. 2014, Article ID 380874, 9 pages, 2014.

[13] J. L. Palacios and J. M. Renom, "Broder and Karlin's formula for hitting times and the Kirchhoff Index," International Journal of Quantum Chemistry, vol. 111, no. 1, pp. 35-39, 2011.

[14] X. Li, Y. Shi, and I. Gutman, Graph Energy, Springer, New York, NY, USA, 2012.

[15] G. Chartrand and P. Zhang, Introduction to Graph Theory, McGraw-Hill, Kalamazoo, Mich, USA, 2004.

[16] I. Gutman, B. Zhou, and B. Furtula, "The Laplacian-energy like invariant is an energy like invariant," MATCH. Communications in Mathematical and in Computer Chemistry, vol. 64, no. 1, pp. 85-96, 2010.

[17] D. Stevanović, A. Ilić, C. Onişor, and M. V. Diudea, "LEL-a newly designed molecular descriptor," Acta Chimica Slovenica, vol. 56, no. 2, pp. 410-417, 2009.

[18] C. Pozrikidis, An Introduction to Grids, Graphs, and Networks, Oxford University Press, Oxford, UK, 2014.

[19] L. Ye, "The energy of a type of lattice," Applied Mathematics Letters, vol. 24, no. 2, pp. 145-148, 2011.

[20] W. Yan and Z. Zhang, "Asymptotic energy of lattices," Physica A. Statistical Mechanics and its Applications, vol. 388, no. 8, pp. 1463-1471, 2009.

[21] D. Cvetković and S. K. Simić, "Towards a spectral theory of graphs based on the signless Laplacian I," Publications de l'Institut Mathématique, vol. 85, pp. 19-33, 2009.

[22] D. Cvetković and S. K. Simić, "Towards a spectral theory of graphs based on the signless Laplacian, II," Linear Algebra and Its Applications, vol. 432, no. 9, pp. 2257-2272, 2010.

[23] D. Cvetković and S. K. Simić, "Towards a spectral theory of graphs based on the signless Laplacian, III," Applicable Analysis and Discrete Mathematics, vol. 4, no. 1, pp. 156-166, 2010.

[24] I. Gutman, "Estimating the laplacian energy-like molecular structure descriptor," Zeitschrift fur Naturforschung-Section A, vol. 67, no. 6-7, pp. 403-406, 2012.

[25] I. Gutman, B. Zhou, and B. Furtula, "The Laplacian-energy like invariant is an energy like invariant," MATCH Communications in Mathematical and in Computer Chemistry, vol. 64, pp. 85-96, 2010.

[26] E. Estrada and N. Hatano, "Topological atomic displacements, Kirchhoff and Wiener indices of molecules," Chemical Physics Letters, vol. 486, no. 4-6, pp. 166-170, 2010.

[27] R. Li, "Lower bounds for the Kirchhoff index," MATCH Communications in Mathematical and in Computer Chemistry, vol. 70, no. 1, pp. 163-174, 2013.

[28] B.-X. Zhu, "The Laplacian-energy like of graphs," Applied Mathematics Letters, vol. 24, no. 9, pp. 1604-1607, 2011.

[29] W. Wang, D. Yang, and Y. Luo, “The Laplacian polynomial and Kirchhoff index of graphs derived from regular graphs," Discrete Applied Mathematics, vol. 161, no. 18, pp. 3063-3071, 2013.

[30] Y. J. Yang, "The Kirchhoff index of subdivisions of graphs," Discrete Applied Mathematics, vol. 171, pp. 153-157, 2014.

[31] Y. J. Yang and X. Y. Jiang, "Unicyclic graphs with extremal Kirchhoff index," MATCH Communications in Mathematical and in Computer Chemistry, vol. 60, no. 1, pp. 107-120, 2008.

[32] A. Mohammadian and B. Tayfeh-Rezaie, "Graphs with four distinct Laplacian eigenvalues," Journal of Algebraic Combinatorics, vol. 34, no. 4, pp. 671-682, 2011.

[33] J. Liu, X. Pan, J. Cao, and F. Hu, "A note on 'some physical and chemical indices of clique-inserted lattices"' Journal of Statistical 
Mechanics: Theory and Experiment, vol. 2014, no. 6, Article ID P06006, 2014.

[34] J.-B. Liu and X.-F. Pan, "Asymptotic incidence energy of lattices," Physica A: Statistical Mechanics and Its Applications, vol. 422, pp. 193-202, 2015.

[35] J.-B. Liu, X.-F. Pan, F.-T. Hu, and F.-F. Hu, "Asymptotic Laplacian-energy-like invariant of lattices," Applied Mathematics and Computation, vol. 253, pp. 205-214, 2015.

[36] J.-B. Liu and X.-F. Pan, "A unified approach to the asymptotic topological indices of various lattices," Applied Mathematics and Computation, vol. 270, pp. 62-73, 2015.

[37] The MathWorks, MATLAB, The MathWorks, Natick, Mass, USA, 2013. 

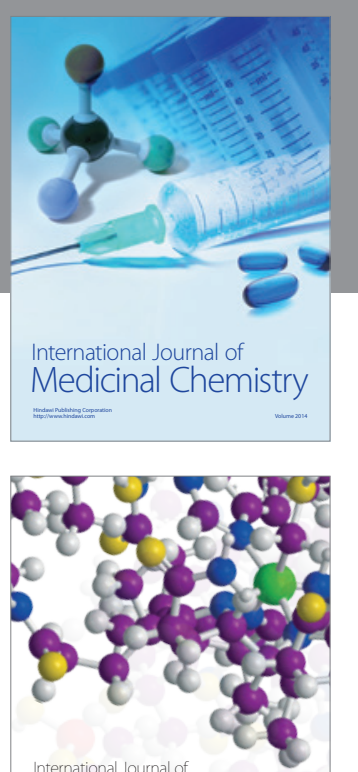

Carbohydrate Chemistry

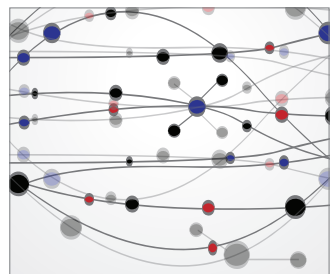

The Scientific World Journal
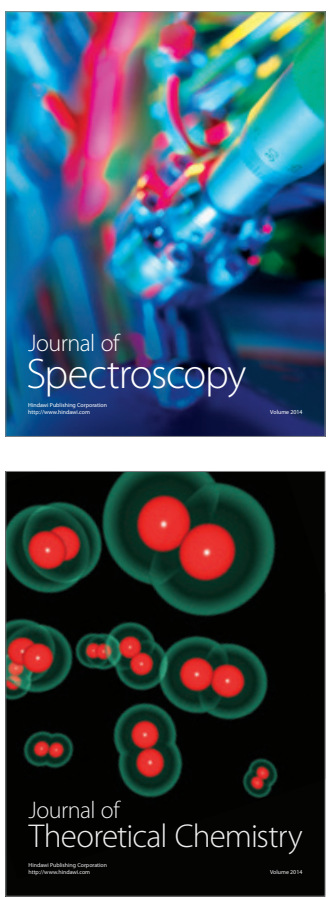
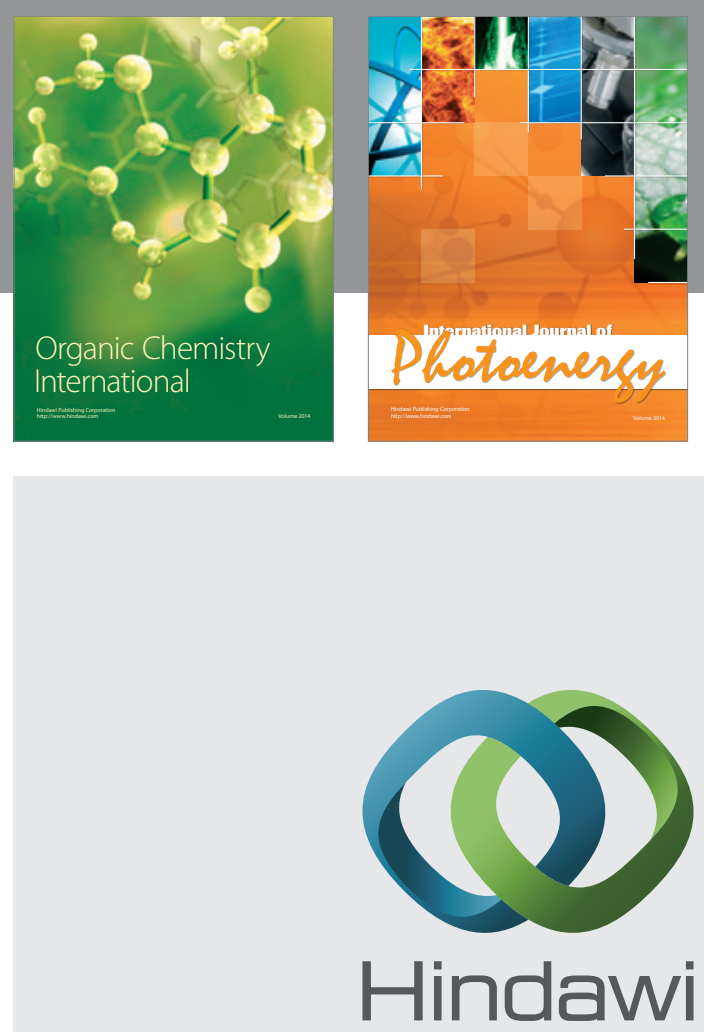

Submit your manuscripts at

http://www.hindawi.com

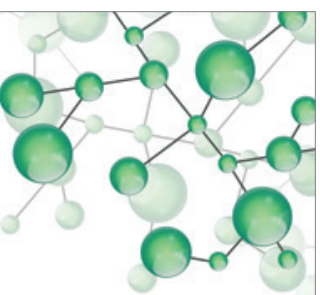

International Journal of

Inorganic Chemistry

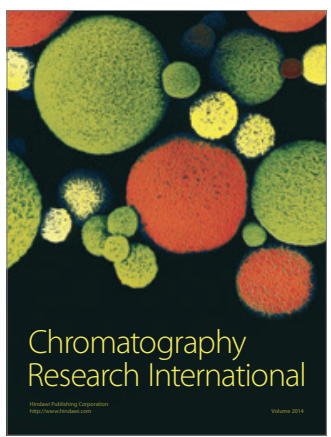

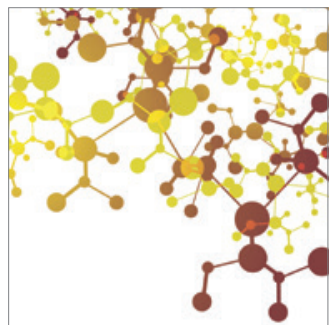

Applied Chemistry
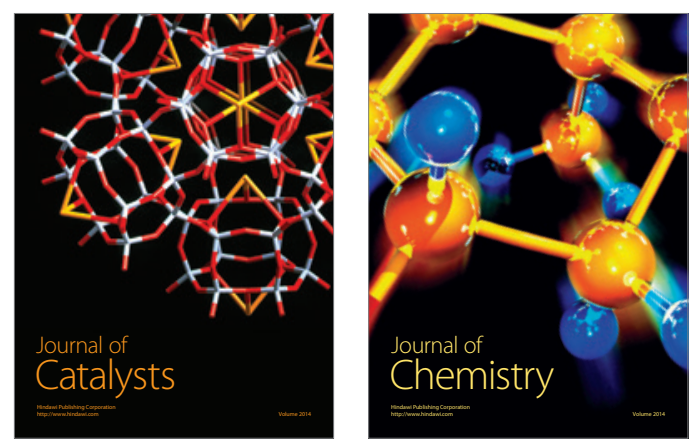
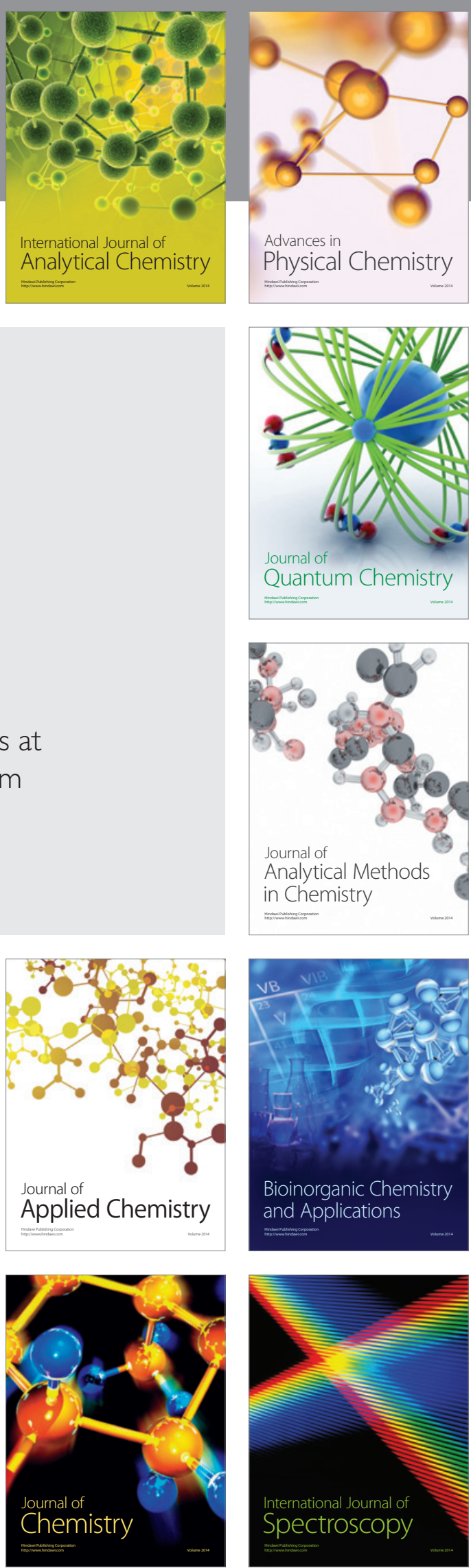